\title{
Left anterior cranial fossa dural arteriovenous fistula ligation using a supra-orbital (eyebrow) craniotomy
}

\author{
Kyle P. O'Connor, BS, and Bradley N. Bohnstedt, MD \\ Department of Neurosurgery, University of Oklahoma Health Sciences Center, Oklahoma City, Oklahoma
}

\begin{abstract}
A 67-year-old male presented to the hospital with a left anterior cranial fossa arteriovenous fistula connecting the anterior ethmoidal artery to the cavernous sinus and superior sagittal sinus. After failed embolization, the patient was taken for a supra-orbital (eyebrow) craniotomy for surgical dissection and clipping of the fistula. An intraoperative angiogram confirmed successful fistula ligation. The patient was discharged without complications.
\end{abstract}

The video can be found here: https://youtu.be/79Pk11SEkJg.

KEYWORDS anterior cranial fossa arteriovenous fistula clipping; supra-orbital (eyebrow) craniotomy; video 